\title{
Application Forum
}

\section{Monitoring Protein Fate during Purification with the Agilent 2100 Bioanalyzer}

\author{
Production-QA/QC
}

Agilent Technologies, Inc., Max Planck Institute of Biochemistry

Claudia Franke and Sabine Suppmann

82152 Martinsried, Germany

\section{Abstract}

Monitoring protein fate with respect to recovery, stability and purity in a fast and reliable manner is fundamental in protein purification. In this Application Note, we present a purification strategy developed for a tag-labeled DNA binding protein, in which the protein fate was monitored with the Agilent 2100 Bioanalyzer. Lab-on-a-chip analysis provides several advantages in quantification, resolution, time, and data handling. Therefore, we have replaced conventional SDS-PAGE with Bioanalyzer analysis.

\section{Introduction}

At the Microchemistry Core Facility of the Max Planck Institute of Biochemistry, we provide an in-house service for recombinant protein production. Our cloning, expression, and purification approaches are very close to a consensus recently reviewed by a number of structural consortia, with immobilized metal affinity chromatography (IMAC) being the most common purification method (1). Although hexahistidine and other affinity tags have simplified protein purification tremendously, there are still a number of challenges remaining, including protein degradation, precipitation, soluble aggregates, and loss on hydrophobic surfaces.

Therefore, an accurate and fast protein sample analysis method is critical for optimizing a protein expression and purification strategy. In this study, we optimized our protein purification strategy for a His6-tagged DNA-binding protein with the help of the Bioanalyzer Protein 230 kit for protein sizing and impurity assessment.

\section{Experimental}

A DNA-binding protein, the protein of interest (POI), was $\mathrm{N}$-terminally fused to His6-Sumo3-tag (2) bound to $\mathrm{Ni}$ beads, and released from the tag and beads by treatment with His-tagged SenP2 protease (3). Bioanalyzer chips have been prepared according to the instructions provided with the Agilent Protein 230 kit.

\section{Protein production}

The expression level as determined by chip analysis (Figure 1) was $51 \%$ of the total, with a relative concentration of $390 \mathrm{ng} / \mu \mathrm{L}$. Relative concentration is related to an internal standard, the upper marker that migrates at $240 \mathrm{kDa}$ (4). We did not include external calibration samples, which is an option provided in the Bioanalyzer Expert software. The protein level was therefore calculated to $30 \mathrm{mg}$ $\mathrm{POI} / \mathrm{g}$ biomass based on this relative protein quantification strategy. We observed an unexpected electrophoretic behavior of the $54-\mathrm{kDa}$ fusion protein with the Protein 230 Kit separating at $\sim 80 \mathrm{kDa}$. This reproducible offset between the expected and observed molecular size could be due to some specific interaction between the protein and the linear polymer used within the Bioanalyzer protein kits. This was observed in previous studies, specifically for glycosylated proteins (5).

\section{Protein purification by affinity chromatography}

In an initial experiment, we typically use half the amount of $\mathrm{Ni}$ beads relative to the target protein in order to prevent background binding. Initially, we purified from $5 \mathrm{~g}$ cells, which corresponds to $150 \mathrm{mg}$ POI (Figure 1). Empirically, $<100 \%$ of protein detected in those SDS buffer-lyzed samples is amenable to purification. We assumed $~ 50 \%$ soluble protein and loaded the lysate supernatant onto $\mathrm{Ni}$ beads, providing $40 \mathrm{mg}$ binding capacity, which corresponds to a $200 \%$ excess of target protein. Table 1 and Figure 2 illustrate protein recovery as determined by Bioanalyzer analysis. Less than half of POI in the total lysate was soluble and recovered in the lysate supernatant. As anticipated from the bead capacity, $45 \mathrm{mg}$ were depleted from the lysate supernatant, and subsequently released by washes, SenP2 on-column cleavage and final imidazole elution. Together with the flow-through, this adds up to $110 \mathrm{mg}$ POI within the lysate supernatant. 


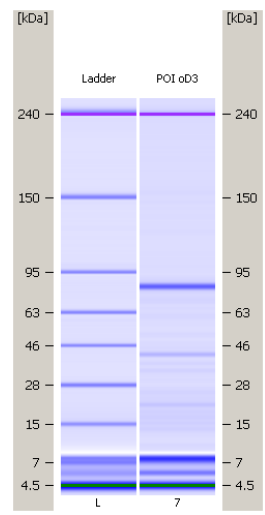

Figure 1. Gel-like image of a 14-230 kDa chip run of cell lysate. One hundred microliters of cells were lyzed in $440 \mu \mathrm{L}, 20 \mathrm{mM}$ Tris $\mathrm{pH} 8.0$ plus $0.25 \% \mathrm{SDS}$ for $5 \mathrm{~min}$ at $95^{\circ} \mathrm{C}$. Based on the relative quantification with the Bioanalyzer, the overall yield for the POI was estimated at $30 \mathrm{mg} / \mathrm{g}$ biomass.

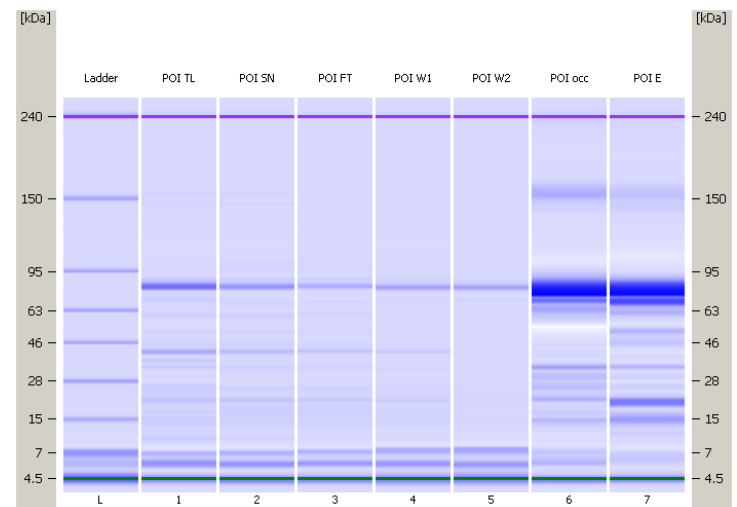

Figure 2. Gel-like image of a Protein $\mathbf{2 3 0}$ chip run monitoring the purification process. From left to right: TL, total lysate 1:10 diluted; SN, lysate supernatant after $30 \mathrm{~min}$ at 20,000 rpm and 1:10 diluted; FT, flow-through 1:10 diluted; W1 and W2, washes 1 and 2; occ, on-column cleavage; E, imidazole eluate. Lysate supernatant from $5 \mathrm{~g}$ cells was loaded to $1 \mathrm{~mL} \mathrm{Ni}$ beads bound for $2 \mathrm{~h}$ at $4^{\circ} \mathrm{C}$, washed twice with $30 \mathrm{~mL}$ washing buffer containing $40 \mathrm{mM}$ imidazole. On-column cleavage was performed in $2 \mathrm{~mL}$ cleavage buffer using 100 units SenP2 protease overnight at $4^{\circ} \mathrm{C}$. Remaining protein was released from $\mathrm{Ni}$ beads with $500 \mathrm{mM}$ imidazole.

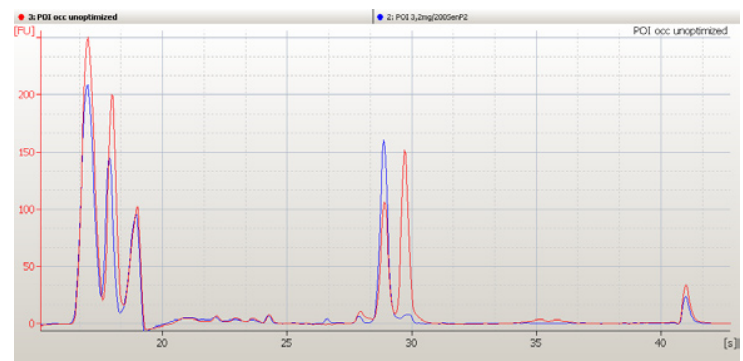

Figure 3. Electropherogram overlay and result table of on-column cleavage samples analyzed with the Protein $\mathbf{2 3 0}$ kit. On-column cleavage of the Histag can easily be monitored. With the optimized protocol, $\mathrm{POI}$ is $78 \%$ pure while tagged $\mathrm{POI}$ is reduced to less than $1 \%$.

\section{Optimization of on-column cleavage}

Although a 100 -fold molar excess of SenP2 protease was used for on-column cleavage, most of the POI released from $\mathrm{Ni}$ beads was still fused to its His6-Sumo3-Tag, probably due to protein aggregation on the beads. When cleaved in solution, fusion protein and tag-free protein interacted very strongly and could not be separated by gel filtration, ion exchange, or any other means. Therefore, the only promising strategy for separating the two forms was to improve on-column tag removal. An excess of beads was used to prevent self-interaction of the POI. An even higher excess of SenP2 protease allowed finding and cleaving its substrate on $\mathrm{Ni}$ beads that were only barely covered with target protein. Finally, the optimal ratio of protein:beads:protease for the best possible on-column cleavage performance was empirically determined as 1:7:100 by monitoring the percentage of cleaved protein versus its tagged precursor (Figure 3). As illustrated in the blue electropherogram (Figure 3), almost all the tagged protein can be cleaved under these conditions. The molecular weight difference of $\sim 10 \mathrm{kD}$ is sufficient to achieve a baseline separation between the tagged and tagless protein.

\section{Final protein purification and QA}

The POI released by SenP2 on-column cleavage at $78 \%$ purity (Figure 3 ) was purified to homogeneity by anion exchange chromatography. Using Bioanalyzer analysis, the final purity of the separated POI was 96.9\% (Figure 4A). Our final quality control of purified proteins also includes analysis by liquid chromatography-mass spectrometry (LC-MS) to assure correct molecular mass and estimate purity based on UV (Figure 4B). Figure 4 shows good correlation between high-performance liquid chromatography (HPLC) separation coupled to UV/electrospray ionization time-of-flight (ESI-TOF) versus electrophoretic separation and fluorescence detection by microfluidics in monitoring the purity of protein samples.

\section{Results and discussion}

We have replaced conventional SDS-PAGE by Agilent 2100 Bioanalyzer chip analysis for several reasons:

- Quantification and resolution. Relative quantification is accurate and allows reliable monitoring of protein fate during purification. Degradation products or contaminating proteins can easily be followed during the course of purification. Double bands, hardly detected in typical Coomassie-stained SDS gels are separated at high resolution. 
Table 1. Protein recovery determined by analysis with the Agilent 2100 Bioanalyzer.

\begin{tabular}{|c|c|c|c|c|}
\hline Sample Rel. & Conc. [ng/pL] & $\%$ Total & Volume [mL] & POI total [mg] \\
\hline Total lysate & 1368.7 & 48.2 & 20 & 274 \\
\hline Lysate supernatant & 551.2 & 38.2 & 20 & 110 \\
\hline Flow-through & 325.1 & 29.9 & 20 & 65 \\
\hline Wash 1 & 435.7 & 48.3 & 30 & 13 \\
\hline Wash 2 & 405.9 & 84.1 & 30 & 12 \\
\hline SenP2 cleavage & 4784.3 & 67.4 & 2 & 10 \\
\hline Imidazole eluate & 9745.4 & 55.2 & 1 & 10 \\
\hline
\end{tabular}

A

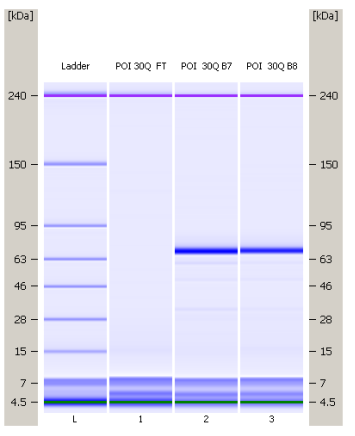

B

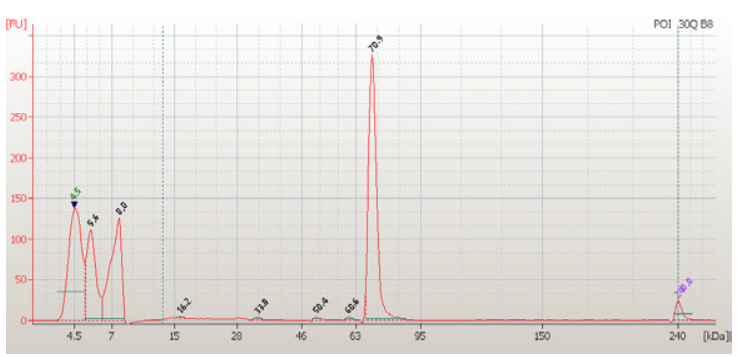

Figure 4. (A) Chip analysis of the POI eluted in a $\mathrm{NaCl}$ gradient on Source $30 \mathrm{Q}$. FT, flow through; $\mathrm{B} 7$ and $\mathrm{B} 8$, protein peak fractions eluted at $45 \mathrm{mM}$ $\mathrm{NaCl}$. Shown are gel-like image, electropherogram, and peak table, indicating that the POI is purified to $\sim 97 \%$. (B) LC-MS of POI measured on Agilent 1100 HPLC-Bruker micrOTOF. UV chromatogram (red trace) returns comparable results to the Bioanalyzer electropherogram.

- Time. Electrophoresis is finished within 20 min. Since data is displayed online, the interpretation of peak tables can be performed immediately. It is unnecessary to stain, destain, scan, and dry gels.

- Data handling. A comparison mode integrates data from different chip runs into one analysis. This allows the reliable comparison of individual protein peaks from different protein samples and experiments. Another helpful tool is the overlay of samples as shown here for SenP2 on-column cleavage efficiency (Figure 3 ).

Because Bioanalyzer protein separation is based on microfluidic capillary gel electrophoresis, relative protein migration times can differ from traditional SDS-PAGE separation. Significant differences were especially observed for proteins that are nonglobular or tend to form higher-level structures (5). Therefore, the first chip analysis of a POI requires some caution. Furthermore, the price per sample is higher than for readymade SDS-PAGE. However, depending on sample throughput, the significantly improved quality of data and faster time to result may justify these drawbacks.

\section{References}

1. Nature Methods, Vol. 5 No 2, February 2008.

2. Kindly provided by Hüseyin Besir, EMBL, Heidelberg.

3. D.Reverter and C.Lima. 2004. Structure 12:1519-1531.

4. Protein Sizing and Quantification with the Agilent Protein 80 and Protein 230 Kits on the Agilent 2100 Bioanalyzer; Agilent Technical Note 5590-5721, May 2010.

5. L. Kelly, P. Barthmaier, Glycoprotein sizing on the Agilent 2100 Bioanalyzer, Agilent Application Note 5989-0332EN, November 2003.

For more information, visit www.agilent.com/chem/labonachip.

Sponsored Paper. BioTechniques 50:333-335 (May 2011) doi 10.2144/000113674

For reprints of this article, contact: biotechniques@fosterprinting.com 